\title{
New 4(3) pair two derivative Runge-Kutta method with FSAL property for solving first order initial value problems
}

\begin{abstract}
A new embedded Two Derivative Runge-Kutta method (TDRK) based on First Same As Last (FSAL) technique for the numerical solution of first order Initial Value Problems (IVPs) is derived. We present an embedded 4(3) pair explicit fourth order TDRK method with a ómallô principal local truncation error coefficient. The stability of the new method is analyzed. The numerical experiments are carried out to show the efficiency of our method in comparison with other existing embedded Runge-Kutta methods (RK) of the same order.
\end{abstract}

Keyword: FSAL technique; IVPs; TDRK methods 